\title{
Analysis of mooring performance parameters of TLP based on AQWA
}

\author{
Aifeng Zhang ${ }^{1, a}$, Meng Wang ${ }^{1, b}$, Miaomiao Yao ${ }^{1, c}$, Shaokang Liu ${ }^{1}$,Jie Wang ${ }^{1}$ \\ ${ }^{1}$ College of Naval Architecture and Ocean Engineering, Dalian Maritime University , Dalian 116026, \\ P.R. China \\ aymin98@163.com, b124476491@qq.com, cymm1300@163.com
}

Keywords: TLP, Hydrodynamic, Pretension, Tendons, AQWA

Abstract. Based on the theory of three-dimensional potential flow and considering the combined wind-wave, the motion response and tendon tension response of Tension leg platform with different pretension and number of tendons are studied by AQWA software. The results from analysis can provide some reference value for the construction of the TLP in the South China sea.

\section{Introduction}

TLP as a new platform has developed rapidly in the United States and Europe. In the decades of operation, the stability of its performance and resistance to harsh environments have been fully affirmed, very suitable for the exploitation of oil resources in the South China Sea[1].However, the current research and development of the tension leg platform is still in the initial stage in China. As the important influent factors, the pretension and the number of tendon are paid more attention in the research and development of TLP.

\section{The theory of three-dimensional potential flow}

The calculation method of the three-dimensional potential flow theory is to put the platform structure as a whole, dividing the mesh on the surface of the underwater platform. The wave load of the platform is calculated by the panel method. The velocity potential satisfies the Laplace equation for the incompressible, irrotational and inviscid flow[2]:

$\nabla^{2} \phi=0$

Bernoulli equation for unsteady flow is:

$$
\frac{\partial \phi}{\partial t}+\frac{1}{2} v^{2}+\frac{p}{\rho}+g z=0
$$

At the free surface, a kinematic boundary condition is written:

$$
\left\{\begin{array}{l}
\frac{\partial \phi}{\partial z}=\frac{\partial \eta}{\partial t}+\frac{\partial \eta}{\partial x} \frac{\partial \phi}{\partial x}+\frac{\partial \eta}{\partial y} \frac{\partial \phi}{\partial y} \\
z=\eta(x, y, t)
\end{array}\right.
$$

The condition at the bottom of the liquid layer is zero normal velocity. The free surface dynamic boundary condition is:

$$
\left\{\begin{array}{l}
\frac{\partial \phi}{\partial t}+\frac{1}{2}|\nabla \phi|^{2}+g \eta=0 \\
z=\eta(x, y, t)
\end{array}\right.
$$




\section{Establishment of Hydrodynamic Model for Tension Leg Platform}

The main model parameters for platform are as below: operating depth $585 \mathrm{~m}$, draft $25 \mathrm{~m}$, displacement $25287.8 \mathrm{t}$ and column outside diameter $16 \mathrm{~m}$. The parameters of the mooring system are shown in Table 1. The hydrodynamic model is established with workbench to the time-domain analysis of platform as shown in Fig. 1. The load is subjected along the positive $x$ direction, taking the one-year return environment conditions in the South China sea. Table 2 is the Environmental conditions of sea.

Table 1 Mooring system parameters

\begin{tabular}{|c|c|c|}
\hline Parameter name & Parameter value & Unit \\
\hline The number of tendon & 8 & $\mathrm{n}$ \\
\hline Tendon in length & 977.8 & $\mathrm{~m}$ \\
\hline Tendon diameter & 0.701 & $\mathrm{~m}$ \\
\hline Tendon wall thickness & 38 & $\mathrm{~mm}$ \\
\hline $\begin{array}{c}\text { Additional inertia force } \\
\text { coefficient }\end{array}$ & 1 & $\mathrm{~N} / \mathrm{A}$ \\
\hline Drag force coefficient & 1.1 & $\mathrm{~N} / \mathrm{A}$ \\
\hline Limit tension & 47000 & $\mathrm{kN}$ \\
\hline Tensile stiffness EA & $2.3 \times 10^{7}$ & $\mathrm{kN}$ \\
\hline Bending stiffness EI & $5.729 \times 10^{6}$ & $\mathrm{kN} \cdot \mathrm{m}^{2}$ \\
\hline
\end{tabular}

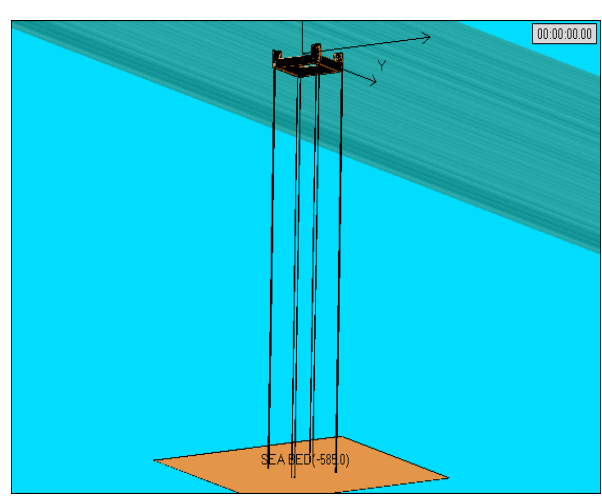

Fig. 1 Time-domain analysis model of TLP

Table 2 Environmental conditions of sea

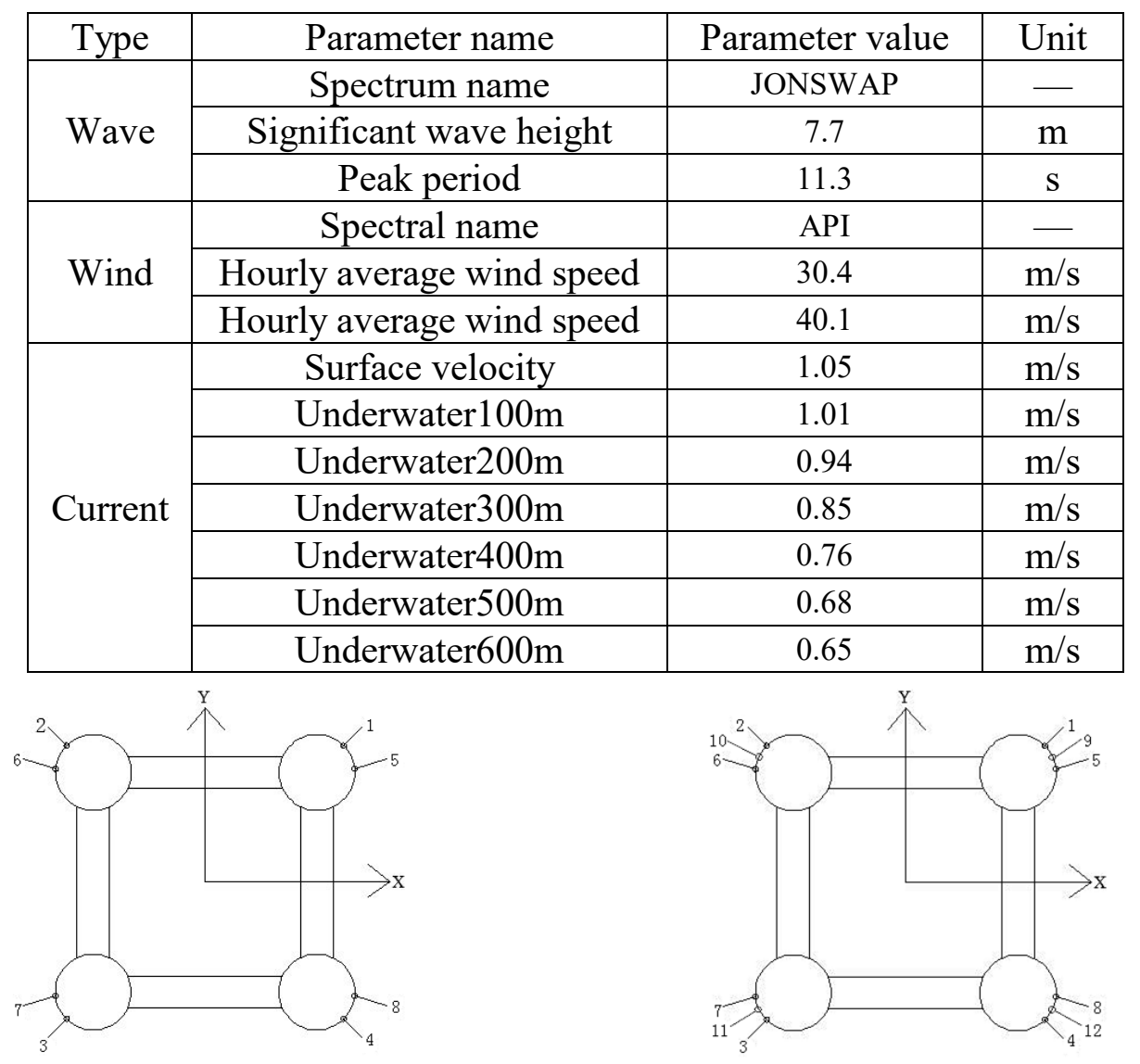

Fig. 2 The distribution of 8 tendons

Fig. 3 The distribution of 12 tendons 


\section{Platform Motion response under different pretension}

Fig. 2 shows the layout of the eight tendons of the platform. The results of platform motion response under different pretension are shown in table 3.

It is clear from the data that with the increase of the pretension, platform motion amplitude has a tendency to decrease because the bigger pretension will increase the tension of the tendons and increase the control of the platform. In the case of heave motion, as the pretension increases, the vertical motion amplitude decreases, but the pretension increases. Keeping the platform draft and buoyancy constant, the load will be reduced, so the heave mean will increase. It is concluded that the motion response of TLP will decrease with the increase of the pretension.

Table 3 Six-degree Motion Response with Different Pretension

\begin{tabular}{|c|c|c|c|c|c|}
\hline Pretension(N) & Degrees of freedom & Minimum & Maximum & Average & Unit \\
\hline \multirow{4}{*}{$2.27 \times 10^{7}$} & Surge & 5.171 & 24.000 & 11.920 & $\mathrm{~m}$ \\
\cline { 2 - 6 } & Sway & 0.156 & 1.566 & 0.842 & $\mathrm{~m}$ \\
\cline { 2 - 6 } & Heave & 1.232 & 1.732 & 1.611 & $\mathrm{~m}$ \\
\cline { 2 - 6 } & Pitch & -0.033 & 0.100 & 0.009 & $\mathrm{deg}$ \\
\cline { 2 - 6 } & Roll & -0.006 & 0.029 & 0.008 & $\mathrm{deg}$ \\
\hline \multirow{4}{*}{$2.53 \times 10^{7}$} & Yaw & -1.246 & 3.280 & 0.433 & $\mathrm{deg}$ \\
\cline { 2 - 6 } & Surge & 4.477 & 22.030 & 10.850 & $\mathrm{~m}$ \\
\cline { 2 - 6 } & Sway & 0.049 & 1.428 & 0.765 & $\mathrm{~m}$ \\
\cline { 2 - 6 } & Heave & 1.393 & 1.809 & 1.715 & $\mathrm{~m}$ \\
\cline { 2 - 6 } & Pitch & -0.029 & 0.090 & 0.007 & $\mathrm{deg}$ \\
\hline & Roll & -0.006 & 0.028 & 0.007 & $\mathrm{deg}$ \\
\hline \multirow{4yyyyy}{*}{$2.78 \times 10^{7}$} & Yaw & -1.231 & 3.102 & 0.395 & $\mathrm{deg}$ \\
\cline { 2 - 6 } & Surge & 3.961 & 20.330 & 9.945 & $\mathrm{~m}$ \\
\cline { 2 - 6 } & Sway & 0.055 & 1.333 & 0.701 & $\mathrm{~m}$ \\
\cline { 2 - 6 } & Heave & 1.539 & 1.897 & 1.813 & $\mathrm{~m}$ \\
\cline { 2 - 6 } & Pitch & -0.025 & 0.081 & 0.006 & $\mathrm{deg}$ \\
\cline { 2 - 6 } & Roll & -0.004 & 0.028 & 0.006 & $\mathrm{deg}$ \\
\hline & Yaw & -1.187 & 2.944 & 0.362 & $\mathrm{deg}$ \\
\hline
\end{tabular}

\section{The tendon tension response under different pretension}

For comparing the tension response results of the tendon under different pretension, Figures 4,5 indicate the simulation curve for No.7,8 tendon. As shown in Figure 2, No.7 and No.8 tendon are on the $y$-axis symmetry, the data is representative.

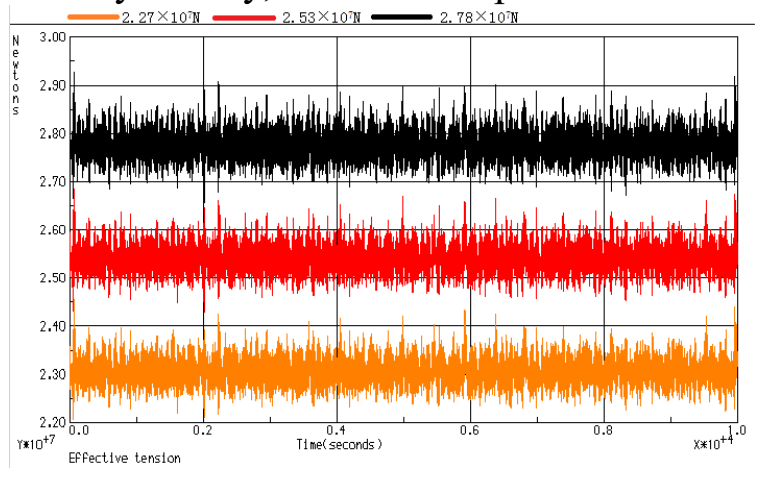

Fig.4 Tension response of No. 7 tendon

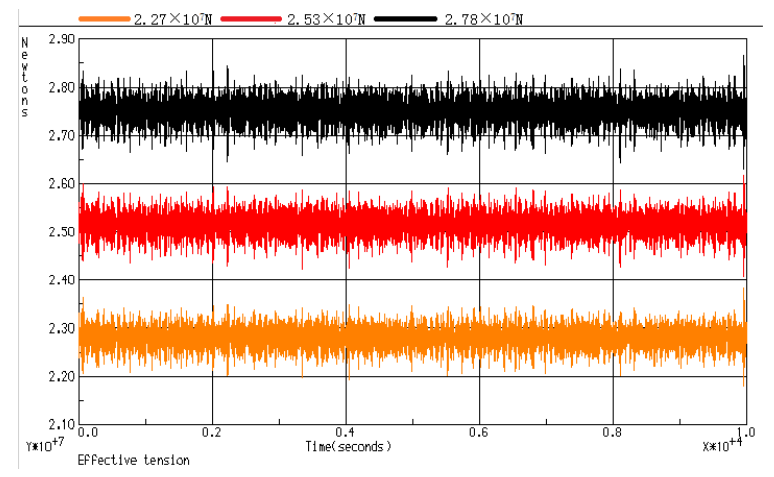

Fig. 5 Tension response of No. 8 tendon

With the increase of pretension, the tension extremity and motion amplitude of platform are obviously enlarged, which inevitably increases the risk of tendon rupture[3]. So increasing the pretension in the design needs to recalculate the safety factor of the tendons of extreme environments. 


\section{Platform motion response with different numbers of tendon}

The traditional TLP tendons number by 8 tendons and 12 tendons, the number of different tendons must have an effect on the movement of the platform[4]. The platform motion and tendon tension response results are calculated for these two different tendons arrangement. Fig. 3 is the distribution of 12 tendons. Table 4 shows the motion response results with different number of tendons.

Table 4 Motion Response with Different Number of Tendons

\begin{tabular}{|c|c|c|c|c|c|}
\hline Total number of tendons & Degrees of freedom & Minimum & Maximum & Average & Unit \\
\hline \multirow{4}{*}{8 tendons } & Surge & 4.477 & 22.030 & 10.850 & $\mathrm{~m}$ \\
\cline { 2 - 6 } & Sway & 0.049 & 1.428 & 0.765 & $\mathrm{~m}$ \\
\cline { 2 - 6 } & Heave & 1.393 & 1.809 & 1.715 & $\mathrm{~m}$ \\
\cline { 2 - 6 } & Pitch & -0.029 & 0.090 & 0.007 & $\mathrm{deg}$ \\
\cline { 2 - 6 } & Roll & -0.006 & 0.028 & 0.006 & $\mathrm{deg}$ \\
\cline { 2 - 6 } & Yaw & -1.231 & 3.102 & 0.395 & $\mathrm{deg}$ \\
\hline \multirow{4}{*}{12 tendons } & Surge & 9.197 & 24.040 & 13.860 & $\mathrm{~m}$ \\
\cline { 2 - 6 } & Sway & 0.175 & 1.328 & 0.762 & $\mathrm{~m}$ \\
\cline { 2 - 6 } & Heave & 1.010 & 1.458 & 1.350 & $\mathrm{~m}$ \\
\cline { 2 - 6 } & Pitch & -0.018 & 0.084 & 0.005 & $\mathrm{deg}$ \\
\cline { 2 - 6 } & Roll & -0.002 & 0.017 & 0.005 & $\mathrm{deg}$ \\
\cline { 2 - 6 } & Yaw & -0.664 & 2.788 & 0.392 & $\mathrm{deg}$ \\
\hline
\end{tabular}

The results from Table 4 indicate that the range of motion of the platform under 12 tendons can be reduced. In addition, the movement of the platform in the three vertical directions of pitching, rolling and heaving decreases. But the horizontal displacement of the platform will increase when the buoyancy remains unchanged and the whole weight of platform is increasing with the tendon numbers. It is possible to lead to the pretension of the tendon reduced and tendons weakened control for the platform.

\section{The tendon tension response with different numbers of tendon}

The tension response of the tendons for the No. 7 and No. 8 under different tendons is shown in Figures 6 and 7.

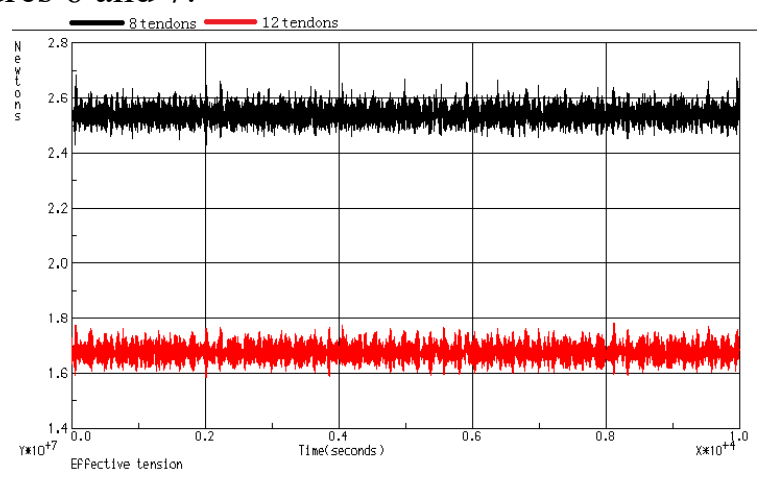

Fig. 6 Tension response of No. 7 tendon

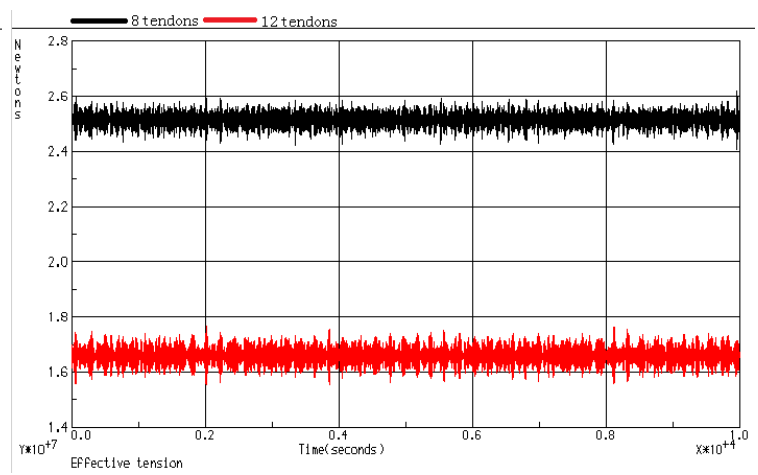

Fig. 7 Tension response of No. 8 tendon

The pretension and tension response of each tendon were significantly smaller under a tension leg platform of 12 tendons, and the extra four tendons can effectively share the tension of other tendons. From other results of tendon, the average tension under 12 tendons is reduced, but the range of changes in tension is smaller. 


\section{Conclusion}

The following conclusions can be drawn from the above simulation results.

The motion response of TLP is larger in the horizontal direction and smaller in the vertical direction, which indicates that the platform has the characteristics of flexibility in the horizontal direction and the approximate rigidity in the vertical direction.

The motion of the upper working platform can be effectively controlled with the increase of pretension, which reduces the movement response of the platform, but increases the tension of each tendon and the response range of tension. So the performance requirements of the tendons should be higher.

The increase of the number of tendons will strengthen the control of the upper platform, allowing the six-degree response of the platform to be controlled in a smaller range. Taking the mean of the motion into account, more tendons will both increase the vertical rigidity and horizontal flexibility of the platform while maintaining the same stage draft. In addition, the added tendon can effectively share the tension of other tendons, so that each tendon tension significantly reduced. It should be noted that the response range of tendon tension changed little and economy of the platform decreased at the same time.

\section{Acknowledgements}

This work was financially supported by "the Fundamental Research Funds for the Central Universities"(3132016339).

\section{References}

[1] Zhanbin Meng, Mu Li and Shugeng Yang: China Offshore Platform 28(4), (2013), p.1-5. (In Chinese).

[2] Yuanli Li, in: Hamics of offshore structures. p.9-36, p.155-192. South China University of Technology Press (1999) (In Chinese).

[3] Ge Liu: Structure design and hydrodynamic analysis of a new type of extended tension leg platform, Ocean University of China, (2013) (In Chinese).

[4] Linfeng Song, Liping Sun, Junlong Zhao, et al: Shipbuilding of China 53(4), (2012), p.7-8. (In Chinese). 